\title{
On Dissipative Radiative MHD Boundary Layer Flow in a Porous Medium Over a Non Isothermal Stretching Sheet
}

\author{
P. Vyas and N. Srivastava $\uparrow$ \\ Department of Mathematics, University of Rajasthan, Jaipur-302055, India \\ †Corresponding Author Email: nupur2209@gmail.com
}

(Received August 22, 2010; accepted July 4, 2011)

\begin{abstract}
The paper aims at investigating the effects of Ohmic and viscous dissipations on the steady two-dimensional radiative boundary-layer flow of an incompressible, viscous, electrically conducting fluid caused by a linearly stretching sheet placed at the bottom of fluid saturated porous medium in the presence of uniform transverse magnetic field. The radiative heat flux is assumed to follow Rosseland approximation. The governing system of partial differential equations are converted to ordinary differential equations by using the similarity transformations, which are then solved numerically using shooting method with fourth order Runge-Kutta scheme. The dimensionless temperature distribution is computed for different thermo-physical parameters and presented graphically. The temperature gradient at the sheet and skin friction coefficient are derived numerically and presented through graphs.
\end{abstract}

Keywords: Numerical study, Boundary layer, Stretching sheet, Porous medium, Dissipation, Thermal radiation, MHD, Convective heat transfer.

\section{NOMENCLATURE}

$\begin{array}{ll}\text { Bo } & \text { magnetic field intensity } \\ c & \text { stretching sheet parameter } \\ c_{\mathrm{p}} & \text { specific heat at constant pressure } \\ \mathrm{D} & \text { constant } \\ \mathrm{Ec} & \text { Eckeret number } \\ \mathrm{k}^{*} & \text { mean absorption coefficient } \\ \mathrm{K} & \text { permeability of porous medium } \\ \ell & \text { characteristic length } \\ \mathrm{M} & \text { Hartmann number } \\ \mathrm{N} & \text { Radiation parameter } \\ \mathrm{Pr} & \text { Prandtl number } \\ \mathrm{q}_{\mathrm{r}} & \text { radiation heat flux } \\ \mathrm{T} & \text { fluid temperature } \\ \mathrm{T} \infty & \text { uniform temperature of the } \\ & \text { ambient fluid }\end{array}$

\section{INTRODUCTION}

Viscous dissipation amounts to local production of thermal energy through the mechanism of viscous stresses. This effect is encountered in both the viscous flow of clear fluids and the fluid flow within the porous medium. Although viscous dissipation effect is considered as a "weak" effect when compared with its other counterpart effects but in many situations it has to be reckon with. The viscous heating aspects in fluids were investigated for its practical interest in polymer industry and the problem was invoked to explain some

$\begin{array}{ll}\text { Tw } & \text { wall temperature } \\ \mathrm{u}, \mathrm{v} & \text { velocity components } \\ \mathrm{x}, \mathrm{y} & \text { Cartesian coordinates } \\ \text { Greek } & \text { Symbols } \\ \psi & \text { stream function } \\ \eta & \text { similarity parameter } \\ \lambda & \text { permeability parameter } \\ \kappa & \text { uniform thermal conductivity } \\ \nu & \text { kinematic viscosity } \\ \rho & \text { density of fluid } \\ \sigma^{*} & \text { Stephan Boltzman constant } \\ \sigma & \text { electrical conductivity } \\ \mu & \text { coefficient of viscosity } \\ \theta & \text { dimensionless temperature }\end{array}$

rheological behavior of silicate melts. The flow studies with viscous heating aspects of viscous fluids demonstrating temperature dependent properties are of immense significance in basic sciences and in contemporary industrial technology such as tribology, instrumentation, food processing, lubrication, polymer manufacturing and many others. Gebhart (1962) came out with observations that devices which operate at high rotational speeds or which are subject to large decelerations experience significant viscous dissipation effect. The effect is felt prominently in strong 
gravitational fields and in processes wherein the scale of the process is very large, e.g. on larger planets, in large masses of gas in space and in geological processes in fluids contained in various bodies. It is pertinent to record that even if viscous dissipation effect is quantitatively negligible in some cases its qualitative effect is significantly observed. The works of Gebhart (1962), Gebhart et al. (1969), Nield (2000) and Magyari et al. (2003), Rees et al. (2003) shed a light on the importance of viscous heating.

In recent years, many researchers have shown interest in the study of boundary-layer flows of viscous fluids over a stretching sheet simply because boundary-layer behavior on a moving continuous solid surface is an important type of flow encountered in several engineering processes. An example of a moving continuous surface is a long thread travelling between a feed roll and a wind-up roll, or a polymer sheet or filament extruded continuously from a die. Investigation of transport processes in porous media have been undertaken with utmost interest due to wide array of applications cutting across different realms such as geothermal engineering, underground disposal of nuclear waste, chemical reactors, heat exchangers and more. Pop et al. (2001), Nield et al. (2006) have presented relevant comprehensive texts.

Sakiadis (1961) was the first to study the flow in the boundary-layer on a continuous solid surface. He considered the boundary layer flow over a flat surface moving with a constant velocity and formulated a boundary layer equation for two-dimensional, axisymmetric flows. Due to entrainment of ambient fluid, this phenomenon represents a different type of boundary-layer problem having solution substantially different from that of boundary-layer flow over semiinfinite flat plate. Crane (1970) extended the work of Sakiadis by considering a moving strip, the velocity of which is proportional to the distance from the slit and obtained closed form exponential solution. Subsequently, many investigators taking the advantage of simplicity of geometry and its exact solution attempted the problem with variety of assumptions. Gupta and Gupta (1977) investigated the heat and mass transfer over an isothermal stretching sheet with suction or blowing. Arunachalam et al. (1978) considered the thermal boundary-layer in liquid metals with variable thermal conductivity for a class of flow where the potential velocity is a power of the distance along a stationary wall. Grubka et al. (1985) analyzed the phenomena with prescribed wall temperature and also with prescribed heat flux and presented their solutions in terms of Kummer's function. Chen et al. (1988) investigated the problem for visco-elastic fluid of Walter's liquid B model subject to power law heat flux. They also obtained their solutions in terms of Kummer's function. Vajravelu et al. (1993) addressed the problem taking the effects of viscous dissipation and internal heat generation into account. Chauhan et al. (1995) examined heat transfer in MHD viscous flow due to stretching of boundary in the presence of naturally permeable bed. Anderson et al. (1998) examined the flow of viscous ferro-fluid over a stretching sheet in the presence of a magnetic dipole to explore the effects of the magneto-thermo-mechanical interaction on skin friction and heat transfer. The problem of linearly stretching sheet was generalized to one that stretches with a power-law velocity by Afzal et al. (1980), Kuiken (1981) and Banks (1983). Chiam (1982) investigated the flow of micropolar fluid over a stretching sheet. Chiam (1995) also investigated the case of a sheet stretching with a power-law velocity and having a variable magnetic field of a special form. Boutros et al. (2006) studied two-dimensional boundary-layer stagnation point flow towards a heated stretching sheet placed in a porous medium using Lie group method. All the above reported investigations were limited to flow and heat transfer without taking radiation into account.

Heat transfer together with radiation from the outer surface of a heated body embedded in a fluid-saturated porous medium finds several applications in geophysics and engineering. Cheng et al. (1977) were the first to present an analysis for the natural convection flows about a heated impermeable surface embedded in fluidsaturated porous media to model the heating of ground water in an aquifer by dike. However, thermal radiation at high temperatures significantly affects the heat transfer and the temperature distribution in the boundary-layer flow of participating fluid. In fact, depending on the surface properties and geometry of the solid, the radiation has dominant impact on flow and heat transfer in porous media. The effect of radiation in thermal regime in porous medium has got wide applications, such as, waste heat storage in aquifers, gasification of oil shale which is of interest on combined convection since fluid is pumped into porous region. In fact, in the case of gasification, large temperature gradient exists in the vicinity of the combustion regime making radiation effect dominant. Raptis (1998) studied radiative micropolar fluid flow past a continuously moving plate. The radiative heat transfer studies are very important in space technology and high temperature processes. But, unfortunately very little has been reported about the effects of radiation on the boundary-layer in porous media. However it is interesting to record that porous medium absorbs/emits radiation that is transferred to or from a fluid. The fluid can be regarded to be transparent to radiation, because the dimensions for the radiative transfer among the solid elements of porous structure are much less than the radiative mean free path for scattering or absorption in the fluid (Howell(2000)). Furthermore, it is pertinent to record that contrary to conduction and convection heat transfer by thermal radiation is a complex phenomenon to account for. Actually fluid radiation studies are confronted with a few difficulties making the things complex and cumbersome. In radiative heat transfer, the prediction of fluid absorption is a tedious task because the radiation is absorbed/emitted not only at system boundaries but also in the interior of the system. Furthermore, the absorption coefficients of the absorbing/emitting fluids are, in general, strongly dependent on wavelength. The computational procedure gets difficult with the presence of radiation term in the energy equation making the equation highly non linear. In view of all these challenges in radiative studies, the effect of radiation on convective flow has been undertaken with reasonable simplifications. Excellent literature on radiation is available in the well presented texts by Sparrow et al. (1970), Özisik (1973) and 
Howell (2000). Using the Roselsand approximation, Plumb et al. (1981) examined the effect of horizontal cross flow and radiation on natural convection from vertical heated surface in saturated porous media. Whitaker (1980) discussed radiative heat transfer in porous media. Tong et al. (1983) proposed two flux models and the linear anisotropic scattering model to predict the radiant heat flux in light weight fibrous insulation. Ali et al. (1984) studied the interaction of natural convection with the thermal radiation in a laminar boundary layer flow over an isothermal, horizontal flat plate. Bakier et al. (1996) investigated the thermal radiation effect on mixed convection from horizontal surfaces in saturated porous media. Ibrahim et al. (1990) investigated mixed convection radiation interaction in boundary-layer flow over a horizontal surface. Hossain et al. (1997) examined radiation effect on free convection of fluid along a heated inclined flat surface maintained at uniform temperature placed in a saturated porous medium. Yih (2001) conducted a study of radiation effect on the mixed convection flow of an optically dense viscous fluid adjacent to an isothermal cone embedded in a saturated porous medium. Al-Odat et al. (2005) presented an analysis on influence of radiation on mixed convection over a wedge in a nonDarcy porous medium. Mukhopadhyay (2009) examined radiation effects on boundary layer flow and heat transfer of a fluid with variable viscosity along a symmetric edge. Pal et al. (2010) investigated heat and mass transfer in MHD non-Darcian flow of a micropolar fluid over a stretching sheet embedded in a porous media with non-uniform heat source and thermal radiation. Vasu et al. (2011) examined the effect of radiation and mass transfer on the transient free convection flow of a dissipative fluid. Prasad et al. (2011) employed finite difference method to examine the radiative free convection flow past an impulsively started vertical plate with variable heat and mass flux.

Magnetoconvection is of considerable interest owing to its frequent occurrence in various realms of industrial technology and geothermal applications, liquid metal fluids, MHD power generation system, high temperature plasmas applicable to nuclear fusion energy conversion. MHD has also been found very useful in controlling boundary-layer transition in the case of subsonic and supersonic flow of gases. Duwairi et al. (2006) presented numerical investigations of magnetohydrodynamic natural convection heat transfer from radiative vertical porous surface. Suneetha et al. (2011) investigated thermal radiation effects on MHD flow past an impulsively started vertical plate in the presence of heat source/sink by taking viscous dissipation into account.

In this communication we wish to study the MHD boundary-layer flow in a porous medium with radiative and dissipative effects. A suitable numerical technique is used to solve the nonlinear energy equation.

\section{Mathematical ANAlysis}

Let us consider the steady, dissipative, MHD twodimensional boundary-layer flow over a linearly stretching sheet placed at the bottom of the fluid saturated porous medium in the presence of radiation. A Cartesian system is used with $\mathrm{x}$-axis is along the sheet and y-axis is normal to it. The sheet is stretched linearly by applying two equal and opposite horizontal forces so that the position of the origin is unaltered. The fluid is considered to be viscous, incompressible, electrically conducting, Newtonian, optically dense and without phase change. We assume that the wall temperature $T_{w}>T_{\infty}$ where $T_{\infty}$ is the uniform temperature of the ambient fluid. It is assumed that both the fluid and porous medium are in local thermal equilibrium and both the fluid and the surroundings are maintained at a constant temperature far away from the sheet. The radiative heat flux in the energy equation is approximated by Rosseland approximation. A magnetic field of strength $B_{0}$ is also applied transverse to the sheet. The induced magnetic field, the external electric field and the electric field due to polarization of charges are neglected while the Ohmic and viscous dissipations are taken into account.

\subsection{Governing Equations and Boundary Conditions}

The governing boundary layer equations can be written as

$\frac{\partial u}{\partial x}+\frac{\partial v}{\partial y}=0$

$u \frac{\partial u}{\partial x}+v \frac{\partial v}{\partial y}=v \frac{\partial^{2} u}{\partial y^{2}}-\frac{v}{K} u-\frac{\sigma B_{0}^{2} u}{\rho}$

$\mathrm{u} \frac{\partial \mathrm{T}}{\partial \mathrm{x}}+\mathrm{v} \frac{\partial \mathrm{T}}{\partial \mathrm{y}}=$

$\frac{\kappa}{\rho c_{p}} \frac{\partial^{2} T}{\partial y^{2}}-\frac{\partial q_{r}}{\partial y}+\frac{\sigma B_{0}^{2} u^{2}}{\rho c_{p}}+\frac{\mu}{\rho c_{p}}\left(\frac{\partial u}{\partial y}\right)^{2}$

The corresponding boundary conditions are

$$
\begin{aligned}
& \mathrm{y}=0: \quad \mathrm{u}=\mathrm{cx}, \quad \mathrm{v}=0 \\
& \mathrm{y}=0: \quad \mathrm{T}=\mathrm{T}_{\mathrm{w}}(\mathrm{x})=\mathrm{T}_{\infty}+\frac{\mathrm{Dx}^{2}}{\ell^{2}} \\
& \mathrm{y} \rightarrow \infty: \quad \mathrm{u}=0 ; \quad \mathrm{T} \rightarrow \mathrm{T}_{\infty}
\end{aligned}
$$

Where the radiation heat flux (Brewster(1972)) is

$$
\mathrm{q}_{\mathrm{r}}=\frac{-4 \sigma^{*}}{3 \mathrm{k}^{*}} \frac{\partial \mathrm{T}^{4}}{\partial \mathrm{y}}
$$

Here the temperature difference within the flow is assumed to be sufficiently small so that $\mathrm{T}^{4}$ may be expressed as a linear function of temperature $\mathrm{T}$, using a truncated Taylor series about the free stream temperature $\mathrm{T}_{\infty}$ to yield

$\mathrm{T}^{4} \cong 4 \mathrm{~T}_{\infty}^{3} \mathrm{~T}-3 \mathrm{~T}_{\infty}^{4}$

\subsection{Similarity Transformation}

Let us introduce the following similarity transformations 


$$
\psi=\sqrt{\mathrm{cu}} \times f(\eta), \quad \eta=\sqrt{\frac{\mathrm{c}}{v}} \mathrm{y}, \quad \theta(\eta)=\frac{\mathrm{T}-\mathrm{T}_{\infty}}{\mathrm{T}_{w}-\mathrm{T}_{\infty}}
$$

where $\psi$ is the stream function such that

$$
\mathrm{u}=\frac{\partial \psi}{\partial \mathrm{y}}, \quad \mathrm{v}=-\frac{\partial \psi}{\partial \mathrm{x}}
$$

From Eq. (7) and Eq. (8) we get velocity components as

$$
\mathrm{u}=\operatorname{cxf}^{\prime}(\eta) \text { and } \mathrm{v}=-\sqrt{\mathrm{uc}} \mathrm{f}(\eta)
$$

We see that the equation of continuity in view of Eq. (9), is identically satisfied.

The central objective of this paper is to investigate thermal aspects of the problem. In order to solve the energy Eq. (3) the temperature distribution is assumed in the form of similar solution as

$$
\mathrm{T}=\mathrm{T}_{\infty}+\mathrm{D} \frac{\mathrm{x}^{2}}{\ell^{2}} \theta(\eta)
$$

The momentum Eq. (2) and energy Eq. (3) in view of Eqs. (5)- (10) reduce to the following form

$$
\begin{aligned}
& \mathrm{f}^{\prime \prime \prime}+\mathrm{ff}^{\prime \prime}+\mathrm{f}^{\prime 2}-\left(\frac{1}{\lambda}+\mathrm{M}^{2}\right) \mathrm{f}^{\prime}=0 \\
& \frac{1}{\operatorname{Pr}}\left(1+\frac{4 \mathrm{~N}}{3}\right) \theta^{\prime \prime}+\mathrm{f}^{\prime}-2 \mathrm{f}^{\prime} \theta+\mathrm{M}^{2} \mathrm{Ecf}{ }^{\prime 2}+\mathrm{Ecf}^{\prime \prime 2}=0
\end{aligned}
$$

with the corresponding boundary conditions

$$
\begin{aligned}
& \eta=0, \quad f=0, f^{\prime}=1, \quad \theta=1 \\
& \eta \rightarrow \infty, \quad f^{\prime}=0, \quad \theta=0
\end{aligned}
$$

where prime denotes differentiation with respect to $\eta$.

Here $M$ is the Hartmann number, where $M^{2}=\frac{\sigma B_{0}^{2}}{\rho c_{p}}$, $\mathrm{N}=\frac{4 \sigma^{*} \mathrm{~T}_{\infty}^{3}}{\kappa \mathrm{k}^{*}}$ is the radiation parameter and $\operatorname{Pr}=\frac{\rho \cup \mathrm{c}_{\mathrm{p}}}{\kappa}$ is the Prandtl number. Also $E c=\frac{c^{2} \ell^{2}}{c_{p} D}$ is the Eckeret number and $\lambda=\frac{\mathrm{cK}}{\mathrm{v}} \quad$ is the Permeability parameter.

\subsection{Solution Procedure}

The solution of eq. (11) is assumed to be of the form

$$
f(\eta)=A+B e^{-s \eta}
$$

where the constants A, B and s are given by

$$
\mathrm{A}=\frac{1}{\mathrm{~s}}, \mathrm{~B}=-\frac{1}{\mathrm{~s}}, \text { and } \mathrm{s}=\sqrt{1+\left(\frac{1}{\lambda}+\mathrm{M}^{2}\right)}
$$

Thus the exact solution of Eq. (9) can be written as

$$
\begin{aligned}
& f(\eta)=\frac{1}{s}\left(1-e^{-s \eta}\right) \\
& f^{\prime}(\eta)=e^{-s \eta}
\end{aligned}
$$

The non-linear boundary value problem given by Eq. (12) with Eqs. (16)-(17) and boundary conditions given by Eq. (13) does not possess closed form analytical solution. Therefore it has been solved numerically by fourth order Runge-Kutta scheme together with shooting method. The computational procedure involved two challenges, firstly determination of $\eta_{\infty}$ i.e. maximum value of $\eta$ for which $\theta(\eta) \rightarrow 0$ as $\eta \rightarrow \infty$. Secondly, in order to employ shooting method, appropriate guesses of $\theta^{\prime}(0)$ are required so that the condition at the other end is satisfied.

The secant method of iteration was used to search for missing $\theta^{\prime}(0)$. The values of $\eta_{\infty}$ for which $\theta(\eta)$ decays exponentially to zero for different set of values of parameters $\lambda, M, N, E c$ and $\operatorname{Pr}$ was chosen after some preliminary investigation. A grid independence study was carried out to examine the effect of the step size $\Delta \eta$ and the edge of the boundary layer $\eta_{\infty}$ on the solution in the quest for their optimization. The $\eta_{\max }$ i.e. $\eta$ at $\infty$ was so chosen that further changes in it showed little changes (constant till $10^{-6}$ ) in the values of $\theta^{\prime}(0)$ vis a vis boundary condition $\theta(\eta) \rightarrow 0$ as $\eta \rightarrow \infty$ is satisfied.

A step size $\Delta \eta=0.025$ was found to be satisfactory for a convergence criterion of $10^{-6}$ in all the cases and the value of $\eta_{\infty}=70$ was found to be sufficiently large for the velocity to reach the relevant free stream velocity.

The procedure adopted above provides wall temperature gradient at the wall numerically that has been shown graphically.

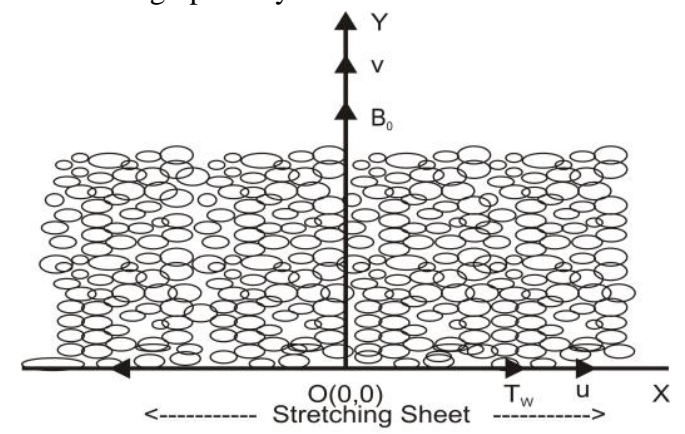

Fig. 1. Physical Model

\section{SKIN FRICTION}

The skin friction $\tau^{*}$ at the wall in the non-dimensional form is given as:-

$$
\tau^{*}=\frac{\tau}{\mu \mathrm{cu} \sqrt{\frac{\mathrm{c}}{\mathrm{v}}}}=\mathrm{f}^{\prime \prime}(0)=-\mathrm{s}
$$




\section{RESUlTS AND DiscusSiON}

In order to get the insight of the phenomenon under study, the profiles for the temperature $\theta(\eta)$ and the wall temperature gradient $\theta^{\prime}(0)$ have been drawn for variable parameter values. Numerous set of values of the parameters central to the study were used to draw profiles, however, selective cases have been reported.

The variations of $\theta(\eta)$ versus $\eta$ have been depicted in Fig. 2 - Fig. 4.

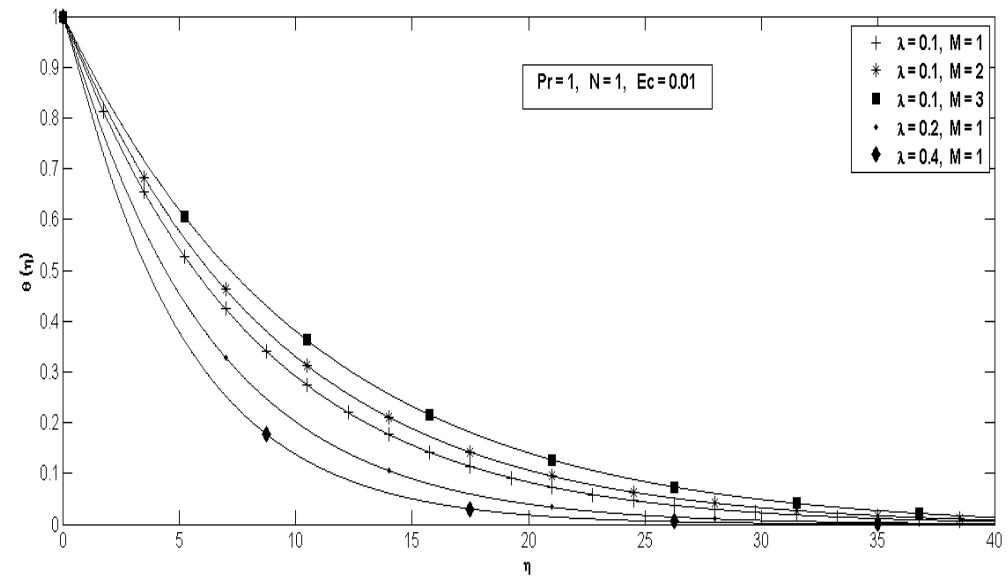

Fig. 2. Temperature distribution versus $\eta$ for varying $\lambda$ and $M$

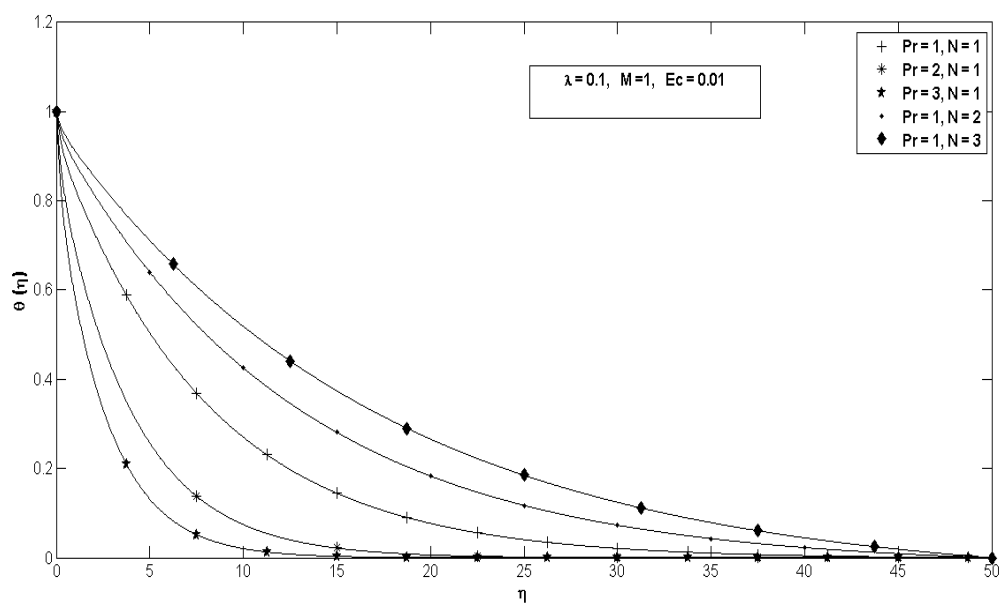

Fig. 3. Temperature distribution versus $\eta$ for varying $\operatorname{Pr}$ and $N$

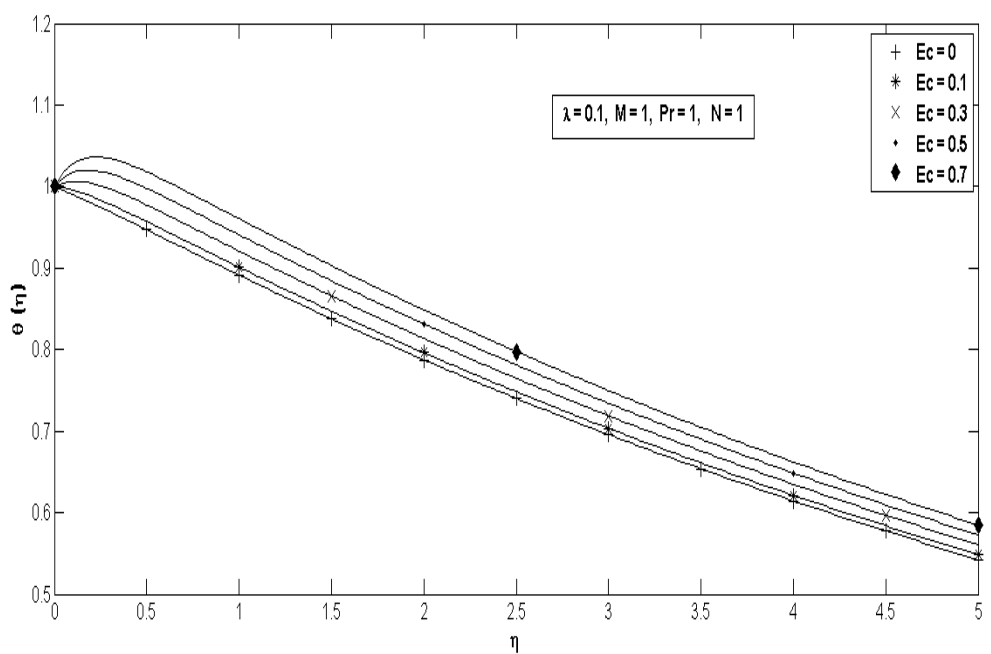

Fig. 4. Temperature distribution versus $\eta$ for varying Ec

Figure 2 displays variation in $\theta(\eta)$ for different values of permeability parameter $\lambda$ and Hartmann number M.
The figure envisages that $\theta(\eta)$ registers increment with the decreasing values of $\lambda$. Here it is worth to note that 
the permeability parameter $\lambda$ is the inverse of Darcian drag force in the porous medium. Thus, larger values of $\lambda$ signifies rather low resistance by the porous medium to cause more ease for fluid trasversal in porous medium. Hence, for larger values of $\lambda$, the thermal boundary layer thickness decays with a physical significance of shorter cooling time. Fig. 2 further reveals that the temperature $\theta(\eta)$ increases with the increasing values of M. Actually, the Hartmann number $\mathrm{M}$ is the measure of the relative importance of Lorentz force to the viscous hydrodynamic force. Thus, larger values of $\mathrm{M}$ indicate stronger magnetic field strength. Furthermore, it is to recall that we have applied transverse magnetic field which retards the flow. This results in hampering of the convective flow with the increasing values of $\mathrm{M}$ and consequently leads to thickening of the thermal boundary layer. In order to understand the effect of Hartmann number (magnetic field parameter), Eq.(2) and Eq.(3) require attention.

The term $\frac{-\sigma \mathrm{B}_{0}^{2} \mathrm{u}}{\rho}$ in Eq. (2) is the Lorentz force which would act in opposite direction of the flow, hence retards the flow. Further the term $\frac{-\sigma \mathrm{B}_{0}^{2} \mathrm{u}^{2}}{\rho \mathrm{c}_{\mathrm{p}}}$ in Eq.

represents Ohmic heating due to electromagnetic work and serves as heat source to cause rise in the fluid temperature. Here it is not out of place to emphasize that the Lorentz force can be used to control the flow of electrically conducting fluid that can serve to assist or oppose the flow (opposing flow and aiding flow).

Figure 3 demonstrates the variation in $\theta(\eta)$ with Prandtl number $\mathrm{Pr}$ and the radiation parameter N. From the very figure we observe that $\theta(\eta)$ registers decrement with increasing values of Pr. Prandtl number physically, signifies the relative importance of momentum diffusion to thermal diffusion in the flow field. In fact dense fluids such as oils enjoy larger Prandtl number values whereas low density fluids such as liquid metals are characterized by low Prandtl number values less than unity. In thermal regime with low Prandtl number (e.g. mercury, $\operatorname{Pr}=0.023$ ), the thermal diffusivity has an upper hand i.e. the heat diffuses at faster rate compared to the momentum. However it should be noted that low Prandtl number fluids exhibit temperature dependent thermal conductivity. On the contrary to it, fossil oil having low thermal conductivity (high Pr values) gives rise to thinner boundary layer. From the fig. 3 it is also observed that $\theta(\eta)$ increases with the increasing values of $\mathrm{N}$. The radiation parameter $\mathrm{N}$ being reciprocal of Stark number (also known as Stephan number) is the measure of relative importance of thermal radiation transfer to the conduction heat transfer. Thus larger values of $\mathrm{N}$ sound dominance of thermal radiation over conduction. Consequently larger values of $\mathrm{N}$ are indicative of larger amount of radiative heat energy being poured into the system, causing rise in $\theta(\eta)$.

Figure 4 depicts the effect of Eckert number Ec on the non-dimensional temperature. It is revealed that $\theta(\eta)$ scores growth with the increasing values of Ec. Eckert number, physically, is a measure of frictional heat in the system. Hence the thermal regime with larger Ec values is subjected to rather more frictional heating causing a source of rise in the temperature. To be specific, the Eckert number, Ec signifies the relative importance of viscous heating to thermal diffusion. Viscous heating may serve as energy source to modify the temperature regime qualitatively. Here a comparison of Fig. 2 and Fig. 4 is interesting. Close examination of the profiles in these figures would reveal the impact of Eckert number, Ec. In Fig. 4 we find that the temperature in the vicinity of the sheet rises considerably (note that at wall $\theta(0)=1$ ) above 1 for larger Ec values. This is due to the frictional heating.

The variation of rate of heat transfer at the sheet $\left(-\theta^{\prime}(0)\right)$ with respect to Prandtl number $\operatorname{Pr}$ is shown from Fig. 5 - Fig. 7.

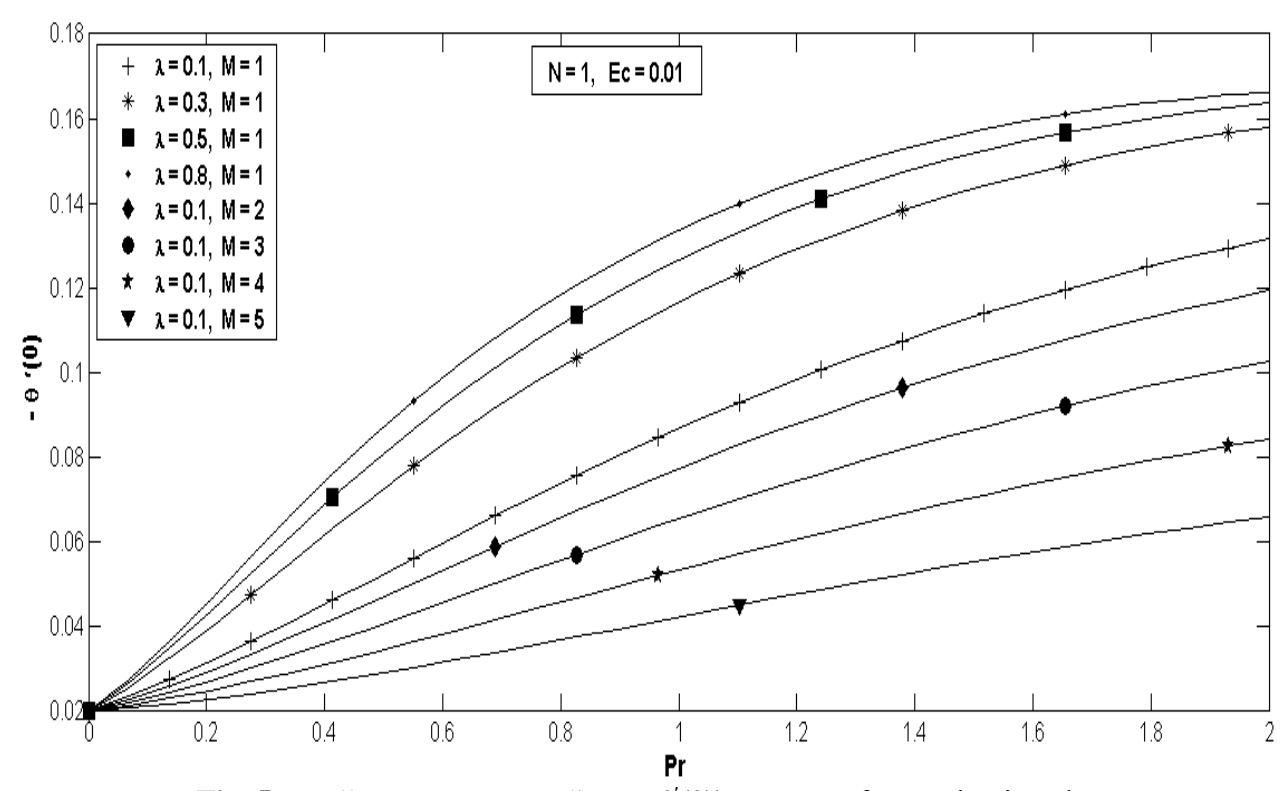

Fig. 5. Wall temperature gradient ( $\left.-\theta^{\prime}(0)\right)$ versus $\operatorname{Pr}$ for varying $\lambda$ and $M$ 
P. Vyas et al. / JAFM, Vol. 5, No. 4, pp. 23-31, 2012.

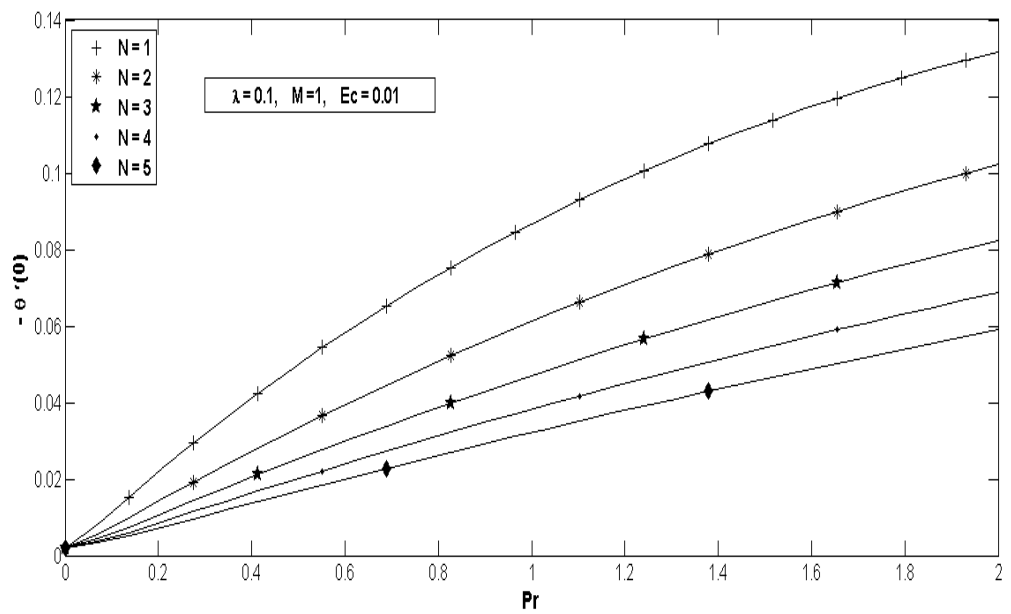

Fig. 6. Wall temperature gradient $\left(-\theta^{\prime}(0)\right)$ versus Pr for varying $N$

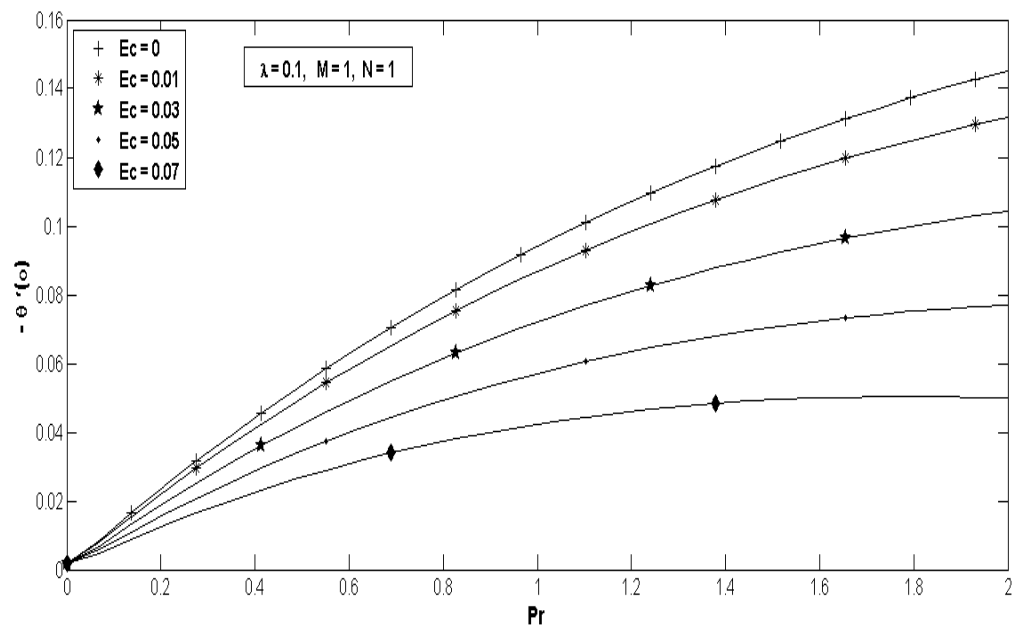

Fig. 7. Wall temperature gradient $\left(-\theta^{\prime}(0)\right)$ versus Pr for varying Ec

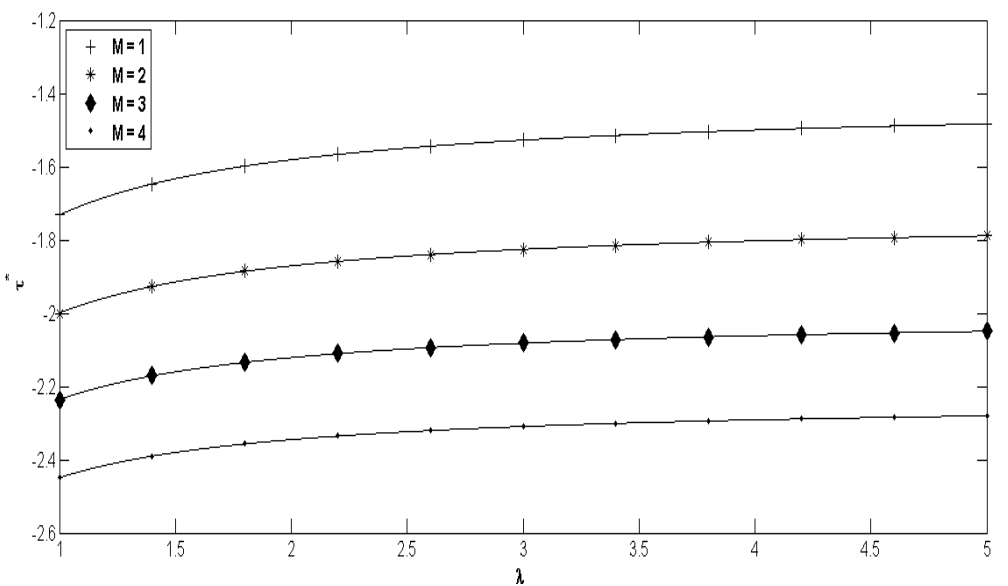

Fig.8. Skin friction coefficient versus $\lambda$ for varying $M$

Figure 5 shows that $\left(-\theta^{\prime}(0)\right)$ increases with an increase in Prandtl number $\operatorname{Pr}$ and also with increase in permeability parameter $\lambda$. On the other hand it decreases with an increase in Hartmann number $M$.

Figure 6 clearly shows that the wall temperature gradient decreases with an increase in Radiation Parameter N.
Figure 7 shows the variation of $\left(-\theta^{\prime}(0)\right)$ for varying values of Eckeret number Ec. It is observed that the temperature gradient at the sheet decreases with an increase in Eckeret number Ec.

Figure 8 shows the variation of skin friction with respect to the permeability parameter $\lambda$ and indicates 
that it decreases with an increase in magnetic field parameter $\mathrm{M}$.

\section{CONCLUSION}

In this study, forced convention is examined including the viscous heating and the magnetic field effects.

1. It is observed that the thickness of the thermal boundary layer, in the vicinity of the stretching sheet increases with an increase in Hartmann number $\mathrm{M}$, Radiation parameter $\mathrm{N}$ and Eckeret number Ec.

2. It is also observed that the thickness of the thermal boundary layer decreases with an increase in permeability parameter $\lambda$ and Prandtl number Pr.

3. Rate of heat transfer at the wall increases with an increase in permeability parameter $\lambda$ and Prandtl number Pr.

4. Rate of heat transfer at the wall decreases with an increase in Hartmann number $M$, Radiation parameter $\mathrm{N}$ and Eckeret number Ec.

Skin friction coefficient decreases with an increase in magnetic field parameter $\mathrm{M}$.

\section{ACKNOWLEDGEMENTS}

The first author (PV) is thankful to University Grants Commission, India, for providing financial support vide grant F.No.: MS - 336/304028/08-09/CRO.

We are also thankful to the learned referee's for their valuable suggestions which led to definite improvement of the paper.

\section{REFERENCES}

Afzal, N. and I.S. Varshney (1980). The cooling ofa low heat resistance stretching sheet moving through a fluid. Warme stoffiibertragung, 14, 289-293.

Ali, M. M. , T.S. Chen and Armaly (1984). Naturalconvection-radiation interaction in boundary layer flow over a horizontal surface. AIAA J, 22(12), 1797-1803.

Al-Odat, M.Q., F.M.S. Al-Hussain and R.A. Damesh (2005). Influence of radiation on mixed convection over a wedge in non- Darcy porous regime. Forseh Ingenieurwes 69, 209215.

Anderson, H. I. and Q.A. Valnes (1998). Flow ofa heated ferrofluid over a stretching sheet in the presence of a magnetic dipole. Acta Meccanica, $128,39-47$.

Aurnachalam, M. and N.R. Rajapppa (1978).Forced convection in liquid metals with variable thermal conductivity and capacity. Acta Mechanica, 31,. 25-31.
Bakier, A. and R.S.R. Gorla (1996). Thermalradiation effect on mixed convection from horizontal surfaces in saturated porous media. Transport in porous media, 23, 357363

Banks, W. M. H. (1983). Similarity solution of theboundary layer equations for stretching wall. J. Mec. Theor appl, 2, 357-392.

Boutros, Y. Z., M.B. Abd-el-Malcek, N.A. Badranand H.S. Hassan (2006). Lie group method of solution for steady two dimension boundary layer stagnation point flow towards a heated stretching sheet placed in a porous medium. Meccanica, 41, 681-691.

Brewster, M. Q. (1972). Thermal radiation Transferproperties, Wiley, New York.

Chauhan, D. S. and P. Vyas (1995). Heat Transfer inMHD viscous flow due to stretching of a boundary in the presence of naturally permeable bed. AMSE Periodicals, Modelling, Measurement \& control B, 60, 17-36.

Chen, M. I. and C.K. Chen (1988). Temperaturefield in non-Newtonian flow over a stretching plate with variable heat flux. Int. J. Heat Mass Transfer, 31, No5, 917-921 .

Cheng, P. and W.J. Minkowyez (1977). Freeconvection about a vertical flat plate embedded in a porous medium with application to heat transfer from a dike. $J$. Geophys Res., 82, 2040-2044.

Chiam, T. C. (1982). Micropolar fluid flow over astretching sheet. ZAMM, 62, 565-568.

Chiam, T. C. (1995). Hydromagnetic flow over asurface stretching with power law velocity. Int. J. Eng. Sci, 33, 429-435.

Crane, L.J. (1970). Flow past a stretching plane. ZAngew Math Phys, 21, 645-647.

Duwairi, H. M., R.A. Damesh and B. Tashtoush(2006). Transient non-Boussinesq magnetohydrodynamic free convection flows over a vertical surface. Int. J. Fluid Mech. Res., 33, 137-152.

Gebhart, B. (1962). Effects of viscous dissipation innatural convection. J.fluid Mech, 14, 225232.

Gebhart, B. and J. Mollendorf (1969). Viscousdissipation in external natural convection flows. J.fluid Mech, 38, 97-107.

Grubka, L. J. and K.M. Bobba (1985). Heat transfercharacteristics of continuous stretching surface with variable temperature. Trans ASME, J. Heat Transfer, 107, 248-250. 
Gupta, P. S. and A.S. Gupta (1977). Heat and masstransfer on a stretching sheet with suction or blowing. Cand. J. Chem Eng., 55, 744-746.

Hossain, M. A. and I. Pop (1997). Radiation effect onDarcy free convection flow along an inclined surface placed in a porous medium. Heat Mass Transfer, 32(4), 223-227.

Howell, J.R. (2000). Radiative Transfer in PorousMedia, In : Vafai K (ed). Transport in Porous Media, NewYork, 663-698.

Ibrahim, F.S. and F.M. Hady (1990). Mixed convection radiation interaction in boundary layer flow over a horizontal surface. Astrophys. Space Sci., 168, 263-276.

Kuiken, H. K. (1981). On boundary layers in fluidmechanics that decay algebraically along stretches of wall that are not vanishingly small. I.M.A.J Appl Math, 27, 387-405.

Magyari, E. and B. Keller (2003). The opposingeffect of viscous dissipation allows for a parallel free convection boundary-layer flow along a cold vertical flat plate. Transp. Porous media, 51, 227-230.

Mukhopadhyay, S. (2009). Effects of radiation and variable fluid viscosity on flow and heat transfer along a symmetric wedge. JAFM, Vol 2, No.2,4, 29-34.

Nield, D. A. (2000). Resolution of a paradoxinvolving viscous dissipation and non linear drag in a porous medium. Transp. Porous media, 41, pp 349-357.

Nield, D.A. and A. Bejan (2006). Convection inPorous media, $3^{\text {rd }}$ ed. . Springer-Verlag, New York.

Özisik, M.N. (1973). Radiative Transfer andInteraction with conduction and convection. Willy, NewYork.

Pal, D. and S. Chatterjee (2010). Heat and Masstransfer in MHD non-Darcian flow of a micropolar fluid over a stretching sheet embedded in a porous media with nonuniform heat source and thermal radiation. Comm. In Nonlinear Science and Numerical Simulation, 15(7), 1843-1857.

Plumb, O. A., J.S. Huenfeld and E.J. Eschbach(1981). The effect. of crossflow and radiation on natural convection from vertical heated surfaces in saturated porous media. In: AIAA $16^{\text {th }}$ thermophysics conference, Palo, Alto, CA, USA, June 23-25.
Pop, I. and D.B. Ingham (2001). Convective HeatTransfer: Mathematical and Computational Modelling of Viscous Fluids and Porous media. Pergamon, Oxford.

Prasad, V. R., Reddy, N. Bhaskar,R. Muthucumaraswamy and B. Vasu (2011).Finite Difference analysis of Radiative freeconvection flow past an impulsively startedvertical plate with variable heat and mass flux.JAFM, Vol 4, No. 1, 7, 59-68.

Raptis, A. (1998). Radiation and free convection flowthrough a porous medium. Int. Comm. Heat Mass Transfer, 25, 289-295.

Rees, D.A.S., E. Magyari and B. Keller (2003).The development of the asymptotic viscous dissipation profile in a vertical free convective boundary layer flow in a porous medium. Transport in porous media, 53(3), 347-355.

Sakiadis, B.C. (1961). Boundary - layer behaviour oncontinuous solid surface: I Boundary layer equations for two dimensional and axisymmetric flow. AI Ch E J 7, 26-28.

Suneetha, S., Reddy, N. Bhaskar and Prasad, V.Ramchandra (2011). Radiation and mass transfer effects on MHD free convective Dissipative fluid in the presence of heat source/sink. JAFM, Vol.4, No.1,7, 107-113.

Sparrow, E.M. and R.D. Cess (1970). Radiation HeatTransfer. Brooks/Cole, Belmont, California.

Tong, T.W. and C.L. Tien (1983). Radiative heat transfer in fibras insulation. Trans. ASME. J. Heat Transfer, 105, 70-81.

Vajravelu, K. and A. Hadjinicolaou (1993). Heattransfer in a viscous fluid over a stretching sheet with viscous dissipation and internal heat generation. Int. comm. Heat mass Transfer, 20 , 417-430.

Vasu, B., Prasad, V. Ramchandra and Reddy, N.Bhaskar (2011). Radiation and mass transfer effects on Transient free convection flow of a dissipative fluid past semi-infinite vertical plate with uniform heat and mass flux. JAFM, Vol.4, No.1,7, 15-26.

Whitaker, S. (1980). Radiant energy transport inporous media. Int Eng Chem Fund, 19, 210218.

Yih, K. A. (2001) Radiation effect on mixedconvection over an isothermal cone in porous media. Heat Mass Transfer 37(1), 5357. 